\title{
Pros and Cons of Adjuvant Interferon in the Treatment of Melanoma
}

\author{
Michael S. Sabel, Vernon K. Sondak \\ University of Michigan Comprehensive Cancer Center, Ann Arbor, Michigan, USA
}

Key Words. Interferon $\cdot$ Melanoma $\cdot$ Adjuvant

\section{LEARNing OBJECTIVES}

After completing this course, the reader will be able to:

1. Define the indication for and administration of IFN- $\alpha$ in the adjuvant treatment of melanoma.

2. Explain the rationale both for and against the use of adjuvant interferon.

3. Discuss areas of ongoing research for adjuvant therapy of melanoma.

CME: Access and take the CME test online and receive one hour of AMA PRA category 1 credit at CME.TheOncologist.com

\section{Abstract}

Should interferon alpha (IFN- $\alpha$ ) be considered the standard of care for the adjuvant therapy of high-risk malignant melanoma? For 2003, it was estimated that $\mathbf{5 1 , 4 0 0}$ cases of invasive melanoma would be diagnosed. The risk of recurrence after surgery is reported to be approximately $60 \%$ for patients with thick primary lesions (T4N0M0, American Joint Committee on Cancer [AJCC] stage IIB) and 75\% for patients with regional nodal metastases (T1-4N1M0, AJCC stage III). The observation that melanoma is susceptible to attack by the host's immune system has resulted in the testing of a remarkably broad spectrum of immunotherapies in

\section{INTRODUCTION}

Over the past several decades, the incidence of melanoma has increased at a faster rate than that of any other solid tumor. In the 1930s, the lifetime risk for a person living in the U.S. to develop melanoma was 1 in 1,500. Currently, that risk is 1 in 74, and for 2003 it was estimated that 51,400 cases of invasive melanoma would be diagnosed [1]. Early recognition and the adjuvant setting. Many of these approaches failed to demonstrate a significant clinical impact, until the use of adjuvant IFN- $\alpha$. Conflicting data from several large, randomized clinical trials resulted in a rapid rise and then decline in the use of IFN- $\alpha$ in the adjuvant setting. This roller coaster has left many clinicians still hesitant to strongly recommend it, and the use of adjuvant IFN$\alpha$ in high-risk melanoma remains controversial. This manuscript reviews the leading arguments for and against its routine use and addresses questions regarding its role in the management of high-risk malignant melanoma. The Oncologist 2003;8:451-458

excision of the primary tumor provide the best opportunity for cure. While efforts to improve early diagnosis through education have resulted in the increased detection of early-stage melanoma, many patients still present with high-risk primary melanomas. Patients with thick primary lesions (T4N0M0, American Joint Committee on Cancer [AJCC] stage IIB) and those with regional nodal metastases (T1-4N1M0, AJCC

Correspondence: Michael S. Sabel, M.D., University of Michigan Comprehensive Cancer Center, 3304 Cancer Center, 1500 East Medical Center Drive, Ann Arbor, Michigan 48109, USA. Telehone: 734-936-5827; Fax: 734-647-9647; e-mail: msabel@umich.edu Received February 13, 2003; accepted for publication June 18, 2003. CAlphaMed Press 1083$7159 / 2003 / \$ 12.00 / 0$ 
stage III) have a reported 5-year survival rate ranging from $30 \%-70 \%$. This dismal prognosis is related to the high failure rates associated with surgical therapy in locally and regionally advanced cases. The risk of recurrence after surgery is reported to be approximately $60 \%$ for stage IIB patients and $75 \%$ for stage III patients [2]. Compounding this has been the lack of effective adjuvant therapy, particularly the limited efficacy of cytotoxic chemotherapeutic agents against melanoma.

A beacon of hope in the treatment of melanoma has long been the observation that melanoma is susceptible to attack by the host's immune system. This has resulted in the testing of a remarkably broad spectrum of immunotherapies, including the use of nonspecific immunostimulants, various approaches to vaccine therapies, and cytokine therapy. Many of these approaches failed to demonstrate a significant clinical impact, and the practitioner had been left with few options in treating high-risk melanoma patients with adjuvant therapy. One exception to this, however, has been the use of adjuvant interferon alpha (IFN- $\alpha$ ).

The interferons are cytokines with diverse immunomodulatory effects on tumor cells. Of the multiple subsets of interferons, IFN- $\alpha$ has been shown to possess a reasonable degree of activity against melanoma. While the precise mechanism of action remains poorly understood, there are multiple antitumor effects of IFN- $\alpha$. These include a direct antiproliferative effect, the enhancement of natural killer cell activity, and the upregulation of tumor antigens and/or HLA class I and class II antigens [3]. Initial phase II clinical studies with IFN- $\alpha$ in metastatic melanoma showed response rates in the $10 \%-20 \%$ range $[4,5]$. These response rates, while encouraging, were not significant enough to lead to its widespread use in the treatment of metastatic melanoma. However, observations that patients with nonvisceral disease were more likely to respond suggested that the use of IFN- $\alpha$ may demonstrate a greater impact in patients with micrometastases [6].

This prompted multiple clinical trials exploring the use of IFN- $\alpha$ as an adjuvant therapy for melanoma in patients at high risk of recurrence. Several of these adjuvant trials had positive results. In 1995, the Eastern Cooperative Oncology Group (ECOG) trial E1684 demonstrated significantly longer relapse-free survival (RFS) and overall survival rates with the use of adjuvant high-dose IFN- $\alpha$. Based on that study, the U.S. Food and Drug Administration (FDA) approved the use of IFN- $\alpha$ for the postsurgical adjuvant therapy of high-risk melanoma. Suddenly this became the standard of care, and the use of IFN- $\alpha$ was widely adopted in the community. Unfortunately, the results of both early and subsequent trials have not been as clear as had been hoped, and the adjuvant use of interferon in the treatment of melanoma remains controversial. This article summarizes the arguments in favor of and against the routine use of adjuvant IFN- $\alpha$ for patients with high-risk melanoma.

\section{Pros: Arguments for Adjuvant IFN- $\alpha$}

\section{The Randomized Trials for High-Dose IFN- $\alpha 2 \mathrm{~b}$ Have Clearly Demonstrated an Improved Disease-Free Survival, and the Majority of Trials Have Demonstrated an Improved Overall Survival}

Trials involving adjuvant IFN- $\alpha$ have involved both low-dose and high-dose regimens. The low-dose IFN- $\alpha$ regimen consists of 2-3 mU administered 2-3 times per week for periods of time ranging from 1 to 3 years. While early results suggested that there may be some effectiveness with the low-dose regimens [7, 8], reanalysis and additional trials have demonstrated no benefit to survival $[9,10]$.

Increasing the IFN- $\alpha$ dose seems to provide an improvement in outcome, as first suggested by Creagan et $a l$. and the North Central Cancer Treatment Group (NCCTG) in their report of a randomized trial of patients at intermediate and high risks of recurrence [11]. In that study, patients were treated with 3 months of IFN- $\alpha 2 \mathrm{a} 20 \mathrm{mU} / \mathrm{m}^{2}$ administered i.m. three times per week. There was a significantly greater disease-free survival rate for node-positive patients and a trend toward improved overall survival in that subset (47\% versus 39\% at 5 years), but this was not statistically significant within this small study (262 patients overall, 160 node positive).

When an even greater dose was used, a clear improvement in survival was noted. Kirkwood et al. examined the use of a very high dose of interferon in a randomized trial for the ECOG, E1684 [12]. This regimen involved an induction phase of IFN- $\alpha 2 b 20 \mathrm{mU} / \mathrm{m}^{2}$ i.v. 5 days a week for 4 weeks followed by a maintenance phase of $10 \mathrm{mU} / \mathrm{m}^{2}$ s.c. 3 days a week for the remainder of a year. That regimen was near the maximally tolerated dose and was quite toxic. The results, however, were notably positive. Patients randomized to the treatment group had significantly better disease-free and overall survival rates compared with the control group. IFN$\alpha 2 b$ therapy resulted in a median RFS time that was longer by 9 months ( 1.72 years for IFN- $\alpha 2 b$ patients versus 0.98 years for observation patients) and produced a $42 \%$ greater 5 year RFS rate (37\% for IFN- $\alpha 2 b$ patients versus $26 \%$ for observation patients). In addition, IFN- $\alpha 2 b$ therapy resulted in a median survival time that was significantly greater by 1 year and produced a $24 \%$ better 5 -year survival rate $(46 \%$ for IFN- $\alpha 2 \mathrm{~b}$ patients versus $37 \%$ for observation patients). Based on those results, IFN- $\alpha 2 b$ was approved by the FDA for the adjuvant treatment of high-risk melanoma.

Following the E1684 trial, an ECOG-coordinated intergroup trial was initiated to provide much-needed verification 
of the overall benefit of adjuvant IFN- $\alpha$ and allow a more precise appraisal of the benefit of therapy in the group of patients with thick, node-negative melanomas. The E1690 trial compared high-dose IFN- $\alpha 2 \mathrm{~b}$ and a 2-year low-dose IFN- $\alpha 2 b$ regimen with observation after complete resection of all known disease [13]. Results of that trial confirmed the disease-free survival advantage for high-dose IFN- $\alpha$ seen in the E1684 trial but not the survival advantage. Advocates of adjuvant interferon therapy point out that this discrepancy is likely due to the result of differences in eligibility criteria and, more importantly, the subsequent availability of postrelapse IFN- $\alpha 2 b$ crossover therapy in the E1690 trial compared with the E1684 trial.

A third trial, Intergroup E1694, compared 1 year of high-dose IFN- $\alpha 2 b$ with 2 years of a vaccine called GMK, containing the ganglioside GM2. Gangliosides are carbohydrate antigens found on the surface of melanoma cells as well as normal cells of neural crest origin and tumor cells of other types. A pilot randomized trial suggested a diseasefree survival benefit in patients who were treated with GM2 plus Calmette-Guerin bacilli (BCG) versus those treated with BCG alone following resection of stage III disease [14]. In May 2000, the independent Data Safety Monitoring Committee of the E1694 trial concluded that the high-dose interferon arm was associated with highly significant greater RFS and overall survival rates and mandated that the study be terminated early and the results disclosed [15]. Compared with the observation arms of the E1684 and E1690 trials, there appeared to be no untoward effects of the GMK vaccine on survival. Therefore, of the four major randomized trials, all four demonstrated improvement in RFS, and three of the four demonstrated improvements in overall survival (although the NCCTG trial did not reach statistical significance). Looked upon as a whole, these results validate the benefit of high-dose interferon for the adjuvant therapy of high-risk, resected melanoma.

\section{The Cost of Treatment with High-Dose Interferon is Comparable with Chemotherapy for Other Malignancies}

In today's world of limited health-care dollars, it is imperative that new therapies be cost-efficient. The costeffectiveness of high-dose IFN- $\alpha 2 b$ for patients with highrisk resected melanoma was assessed based on the results of the original E1684 trial. The cost of 1 year of IFN- $\alpha 2 b$ treatment was estimated to be just under $\$ 29,000$ in that analysis. The cost-effectiveness was found to be comparable with other recognized medical therapies, ranging from $\$ 32,600$ $\$ 43,200$ per year of life saved [16]. A cost-effectiveness analysis of adjuvant interferon in Spain, also based on the E1684 data, demonstrated that its use was within limits established in health economics and was comparable with other interventions in which the cost-effectiveness was acceptable [17].

\section{The Toxicities Associated with Treatment are Worthwhile}

The toxicities associated with IFN $\alpha-2 b$ are significant. In the E1684 trial, treatment was reduced or discontinued in over half the patients, and there were two treatment-related deaths. However, the adjuvant use of IFN $\alpha-2 b$ is supported by the results of a quality-of-life-adjusted survival analysis (Quality-Adjusted Time Without Symptoms and Toxicity, Q-TWiST) [18]. Using the Q-TWiST methodology, patients with stage III melanoma randomized to high-dose interferon in the E1684 trial were found to have more time without symptoms or toxicities than the observation group. The treatment group had a mean of 8.9 months more time without disease relapse and 7 months longer overall survival time compared with the observation group; they experienced an average of 5.8 months of severe treatment-related toxicities. This supports the use of IFN- $\alpha$ as adjuvant therapy. If the quality of life during the time with toxicities is valued more highly than the quality of life during the time after relapse, the group receiving interferon had a significantly greater quality-of-life-adjusted time than did the observation group.

\section{There are No Other Effective Adjuvant Therapies}

The need for adjuvant therapy in the treatment of melanoma is dramatic. Patients with thick primary melanomas $(>4 \mathrm{~mm})$ or melanoma metastatic to the regional lymph nodes have relapse rates of 50\%-90\%. Once distant metastases develop, median survival is only 6 to 9 months. Thus, the development of adjuvant systemic therapy that can reduce the recurrence rate of melanoma has been a critical area of investigation. However, to date, no large randomized trial of adjuvant chemotherapy, radiation therapy, or vaccine therapy has shown a benefit in high-risk melanoma patients.

The use of chemotherapy for the adjuvant therapy of high-risk melanoma has been ineffective. This is not surprising given the poor response rates associated with chemotherapy against metastatic melanoma. Dacarbazine (DTIC) was the first agent to show significant activity in melanoma, and it remains the only chemotherapeutic drug approved on label for the treatment of metastatic melanoma [19]. Combination chemotherapy has demonstrated mildly improved response rates, but has not resulted in a significantly greater survival rate versus DTIC alone in stage IV melanoma. In the adjuvant setting, chemotherapy has been a disappointment [20]. For example, a large, multicenter randomized trial performed by the World Health Organization evaluated 761 patients with Clark's level III-V node-negative truncal melanomas or 
any melanoma with positive nodes [21]. Patients were randomized to receive either DTIC alone, BCG alone, BCG plus DTIC, or observation. After a median follow-up of 41 months, no difference in disease-free or overall survival was detected for any group.

Melanoma vaccines hold the most promise in the adjuvant setting, potentially able to improve disease-free and overall survival rates with minimal toxicities. However, the promise of melanoma vaccines has been hinted at for over 40 years without any established clinical benefit. To date, no large, prospective randomized trial has demonstrated an improvement in overall survival associated with vaccine therapies. Many trials have shown that vaccines can effectively generate a quantifiable specific immune response, but it has not been clearly shown that such a response results in long-term survival benefits. Many questions regarding vaccines remain unanswered, and none are ready for off-protocol use [22].

\section{Cons: Arguments Against the Use of AdJUVANT IFN- $\alpha$}

\section{The FDA Approved IFN- $\alpha$ Too Quickly}

The E1684 trial, which established the adjuvant use of IFN- $\alpha 2 b$, is not without limitations. Patients were not stratified by the number of positive lymph nodes, a recognized prognostic factor. Therefore, it is possible that there may have been an unrecognized imbalance between the treatment and control groups that influenced the outcome. However, the substantial relapse rate associated with highrisk melanoma and the lack of effective adjuvant therapies led to the rapid FDA approval of IFN- $\alpha$. This occurred despite multiple trials showing no overall survival benefit to varying doses of interferon and the lack of a confirmatory trial for high-dose interferon. In addition, the FDA approved 1 year of high-dose IFN- $\alpha 2 b$ as adjuvant therapy for stage IIb as well as stage III disease, despite there not being enough stage IIb patients in the trial to make a statistical analysis of that subgroup.

Despite the benefit demonstrated in the E1684 trial, many patients and physicians did not readily accept the treatment, mostly due to the significant side effects and cost of treatment. The disappointing results from the E1690 trial obviously led to a substantial decline in the use of interferon in clinical practice. The E1694 trial revitalized its use, but this roller coaster has left many clinicians still hesitant to strongly recommend it. In addition, despite the enthusiasm and multiple large controlled studies, there has been no demonstrable rationale of the mechanisms of action of this biologic response modifier [23]. Basic studies are still required to clarify the mechanism of action to better define the role of this drug in the treatment of melanoma. Having an FDA-approved adjuvant treatment available, without clear answers as to the best use of it clinically, precludes many patients from participating in clinical trials necessary to provide those answers.

\section{It is Unclear Which High-Risk Patients Would Most Benefit From Adjuvant Therapy}

When discussing the high-risk melanoma patient, we typically refer to either the patient with a thick primary melanoma or the patient with metastases to the regional node. In the NCCTG trial, there was no effect demonstrated in node-negative patients. Likewise, in the E1684 trial, the beneficial effect was confined to node-positive patients. Patients with thick primary melanomas who were nodenegative accounted for only a small subset (about $11 \%$ of the 280 total patients) of the trial, and within that subset, the treatment group actually fared worse. The small size of that subset makes it impossible to determine whether there was truly a difference in the response of node-positive and node-negative patients to interferon treatment. The E1690 trial showed no benefit to the RFS rate in node-negative patients, while the E1694 trial showed a significant benefit in that population. Taken together, however, of the four major trials, three demonstrated no survival advantage to adjuvant interferon in patients with thick primary melanomas but no lymph node involvement. This makes it much more difficult to recommend the use of adjuvant IFN- $\alpha 2 b$ in that patient population.

Even in node-positive patients, the data are not completely clear. The risk of relapse increases with the number of lymph nodes containing metastatic disease [2]. The E1684 trial was not stratified for the number of positive nodes, but, retrospectively, the greatest RFS benefit appeared to be for patients with one positive lymph node [15]. Both the E1690 and E1694 trials were stratified for 0, 1, 2-3, and 4+ nodes. The E1690 trial showed that, when stratified by the number of nodes, the RFS benefit was only significant in patients who had 2-3 positive lymph nodes. Kaplan-Meier estimates of RFS in the E1694 trial showed no significant benefit in patients with 1, 2-3, or $>4$ involved lymph nodes. This leaves open questions as to which population of high-risk patients would benefit from high-dose interferon. It is important to note that several of those studies were performed prior to sentinel lymph node technology becoming standard of care. Whereas many of the patients in those trials had grossly positive nodes or multiple lymph nodes involved, today we see many patients with micrometastases in the sentinel lymph node only. The role of adjuvant interferon in that population is unknown, and is presently being addressed by the ongoing Sunbelt Melanoma Trial. 
There are Too Many Questions Among the Randomized Trials

Concerns about the E1684 trial, specifically the possible imbalance between the treatment and control groups, were supposed to be addressed in the E1690 trial. As mentioned, however, while there was a greater disease-free survival for patients given high-dose IFN- $\alpha$, the study failed to demonstrate a greater overall survival for patients given either high- or low-dose IFN- $\alpha$. Advocates of interferon therapy argue that the failure of the E1690 trial to validate the E1684 trial was due to crossover therapy, the use of offprotocol high-dose IFN- $\alpha$ by patients in the observation arm after relapse. These crossover data have never been adequately published and this must be considered an unproven hypothesis. With so many differences between the two trials, it is difficult to directly compare the results. However, when a follow-up trial meant to verify early results fails to do so, it is hard to dismiss those results and recommend treatment regardless. Supporters of adjuvant IFN- $\alpha$ state that a third trial, the E1694 trial, confirmed the disease-free and overall survival benefits of high-dose IFN$\alpha 2 \mathrm{~b}$ demonstrated in the E1684 trial. However, that study did not have an observation arm. Therefore, a deleterious effect of the GMK vaccine cannot be ruled out. Such an effect would lead to the appearance of a benefit for IFN- $\alpha$ in that trial when none existed.

Even assuming that the GMK vaccine had no detrimental effect on survival, the E1694 trial is not a pure confirmation of the results of the E1684 trial. As described above, the E1684 trial demonstrated the greatest RFS benefit of high-dose IFN- $\alpha 2 b$ among patients presenting with one positive lymph node. In the E1690 trial, the subset with the greatest RFS benefit, and the only one reaching independent statistical significance, was the group of patients with 2-3 positive nodes. Finally, the E1694 trial demonstrated the greatest benefit, and again the only independently statistically significant benefit, in the node-negative subset. One possible explanation is that the RFS benefit of IFN$\alpha 2 b$ may be stage independent, and the relative risk of relapse may be reduced to a similar degree across subgroups [15]. However, the striking differences among the three trials in regard to which patient population derived the greatest benefit (if any) give pause to strongly recommending high-dose interferon to all high-risk melanoma patients.

\section{The Toxicities are Too Great}

The toxicities surrounding the use of IFN- $\alpha$ are not inconsequential. In the E1684 trial, both treatment delays and dosage reductions were required during therapy, including $50 \%$ of patients during the induction period and $48 \%$ of patients during the maintenance phase. Grade 3 toxicities were seen in $67 \%$ of all treated patients at some time in their therapy, while $9 \%$ had grade 4 toxicities. Serious side effects included fatigue, flu-like symptoms (malaise, fevers, chills, arthralgias), liver function abnormalities (fatal hepatotoxicity has been observed in patients who were not carefully monitored and appropriately dosereduced in the event of liver function test elevations), neutropenia, nausea and vomiting, and psychiatric symptoms including depression and suicide. Careful attention to dose-reduction criteria and liberal use of i.v. fluids, antiemetics, and antidepressants can moderate the side effects. However, the successful administration of highdose IFN- $\alpha 2 b$ adjuvant therapy requires a committed team including oncologists, nurses, pharmacists, social workers, and psychiatrists/psychologists.

Despite the significant toxicities, proponents of IFN- $\alpha$ point to the Q-TWiST analysis described above as support for assuring patients that the significant toxicities of therapy appear justified. However, that is the only study of quality of life with adjuvant IFN- $\alpha$, and there are several problems [20]. First, the analysis was done retrospectively using the collected data from the E1684 trial, without prospective collection of quality-of-life data from participants in the trial. The analysis assigned arbitrary relative values to time with toxicity and time with relapse, rather than assessing the actual quality-of-life valuations of the individual patients in the trial. Also, the improvement in overall survival used in that analysis was not reproduced in the E1690 trial, but the Q-TWiST analysis was never updated. More importantly, the Q-TWiST conclusion is based on the assumption that the quality of life of a patient who has symptoms but is disease-free is valued more highly than that of a patient who has relapsed but has no symptoms. If one assumes that the relative values are equal, then the quality-of-life-adjusted time was not statistically significantly greater for patients receiving interferon than for those in the observation group. Patients certainly are averse to the side effects of IFN- $\alpha$; they regularly refuse the recommendation for treatment and seek alternatives in clinical trials or chose to forego adjuvant therapy altogether.

Another important point to consider is that the studies demonstrating the cost-effectiveness of interferon as adjuvant therapy are based solely on the E1684 trial data. That trial showed a dramatic benefit to the use of adjuvant interferon in both disease-free and overall survival rates. In addition, those analyses used projections of long-term survival, since data were only available for 5 years. Given the improved survival of patients in the control arms in both subsequent high-dose interferon trials and the lack of a demonstrated benefit in the E1690 trial, those analyses may not accurately reflect the economic cost-benefit ratios of using adjuvant high-dose IFN- $\alpha 2 b$. 


\section{There are Other Less Toxic Immunotherapies Right Around the Corner}

Vaccine treatments for patients with high-risk primaries or patients with regional lymph-node involvement remain a promising area of clinical investigation, and may be the next breakthrough in melanoma therapy. Vaccines offer the potential for improving disease-free and overall survival rates with minimal toxicities. Two John Wayne Cancer Institute phase III trials testing an allogeneic polyvalent irradiated whole-cell allogeneic melanoma vaccine are currently under way. Compared with historical controls, the phase II results with this allogeneic vaccine showed significantly better survival rates, and certainly supported phase III testing [24]. Furthermore, a great deal of immunologic correlative data (as much or more than was available when IFN- $\alpha$ entered phase III testing) supports the activity of this polyvalent vaccine [25, 26]. Another allogeneic melanoma vaccine, this one a wholecell lysate, has undergone phase III testing by the Southwest Oncology Group (SWOG) in patients with intermediatethickness, node-negative melanoma (SWOG-9035). The compelling rationale for choosing this vaccine was a low, but definite, level of antitumor activity for the vaccine in advanced disease. The results of the SWOG-9035 trial revealed a trend toward better relapse-free survival in the vaccine arm; this reached statistical significance when all randomized patients were considered [27]. Several other approaches to vaccine therapy are presently in clinical trials. To discover the potential benefits of this novel approach, patients with high-risk melanoma should be encouraged to participate in clinical vaccine trials. However, the use of IFN$\alpha$ outside of clinical trials decreases participation in these trials, and any requirement to compare vaccines with IFN- $\alpha$ in randomized trials (as was done in the E1694 trial) limits the ability to determine the relative benefit of vaccine treatment.

\section{Conclusion}

The use of adjuvant IFN- $\alpha$ in high-risk melanoma patients remains controversial and is likely to remain so until additional clinical trial information becomes available. Is it possible, however, to compare the relevant pro and con arguments and come to some justifiable conclusions? Certainly there seem to be some conclusions with which nearly all melanoma physicians would agree. They are listed below.

- Regardless of one's position on the relative benefit of IFN- $\alpha$ adjuvant therapy, we can and must do better. Clinical trials are urgently needed to address the following issues:

- Are there effective agents that can replace or add to IFN- $\alpha$ in the adjuvant therapy of high-risk melanoma?
- Can we decrease the toxicity of the current highdose regimen without losing efficacy, thereby mitigating some of the contrary arguments to its use?

- Can we shorten the duration of the current highdose regimen without losing efficacy, thereby mitigating some of the contrary arguments to its use?

- Can we do better in selecting which melanoma patients are at highest risk for relapse, thus eliminating the need for adjuvant IFN- $\alpha$ in some patients destined not to benefit from it?

- Regardless of one's position on the relative benefit of IFN- $\alpha$ adjuvant therapy for high-risk melanoma, virtually all clinicians and patients agree that the toxicities of therapy are too high for patients with intermediate risks of recurrence, such as those with lower risk primaries and pathologically negative sentinel lymph nodes. Nonetheless, the risk of recurrence in those patients is sufficiently great to justify adjuvant therapy with low short- and long-term toxicities. Clinical trials in intermediate-risk patients should continue, including trials of less intense IFN- $\alpha$ regimens and also phase II and phase III vaccine trials.

- Regardless of one's position on the relative benefit of IFN- $\alpha$ adjuvant therapy for high-risk melanoma, some informed patients continue to feel that the toxicities of therapy are too high, and they seek alternative therapies. Those patients should not be precluded from participating in well-designed, controlled trials that include a no-treatment control arm.

What then of the more contentious issue of the value of IFN- $\alpha$ in the adjuvant setting for high-risk melanoma? How do the authors of this treatise weigh the relative arguments pro and con?

\section{- Is high-dose IFN- $\alpha 2$ b effective?}

YES. Too many clinical trials demonstrate an improvement in relapse-free survival to deny the fact that high-dose IFN- $\alpha 2 b$ alters the natural history of high-risk melanoma. If the E1694 trial is accepted as a clinical trial that documents a significant impact of high-dose IFN- $\alpha 2 b$ on overall survival, then it is impossible not to conclude that adjuvant therapy with high-dose IFN- $\alpha 2 b$ should be routinely offered to healthy melanoma patients at high risk of relapse.

- Should the E1694 trial be accepted as supporting the benefit of high-dose IFN- $\alpha$ on survival? 
YES. The likelihood that vaccination with a ganglioside antigen can result in a markedly greater risk of early dissemination of and death from metastatic melanoma is so far-fetched as to defy reasonable belief. There is credible evidence that anti-GM2 antibodies, either spontaneous or vaccine induced, are protective against melanoma relapse, including evidence from the E1694 trial itself, but no credible evidence of antibodies inducing an exacerbation of melanoma or any human cancer. It is noteworthy that the strongest public proponents of the "killer vaccine" theory offer no such criticisms of the previous clinical data in support of GM2 vaccination, and indeed, in many cases, continue to test either the GMK vaccine itself or other vaccines containing GM2 in clinical trials in melanoma patients.

- Are there any subsets of high-risk melanoma patients that do not benefit from high-dose IFN- $\alpha$ ?

NO. The most plausible explanation of the available data is that the absolute efficacy of IFN- $\alpha$ is consistent across all subsets of high-risk patients: thick node-negative, microscopic node-positive, and macroscopic node-positive at initial presentation and recurrence. It also seems highly reasonable to extrapolate the available clinical trial data to patients at similar or even higher risks of relapse, such as patients with intermediate-thickness, ulcerated melanomas and negative sentinel nodes and patients with completely resected metastatic melanoma. It must be noted, however, that the subset of high-risk patients with the least clinical trial data is the group of patients that is now most commonly treated with adjuvant IFN- $\alpha$, namely, those patients with a single, microscopically positive lymph node found on sentinel node biopsy. More data on the results of untreated and IFN- $\alpha$-treated patients in this subgroup are sorely needed and would enhance the acceptability of adjuvant treatment by physicians and patients alike.
- Is observation a reasonable alternative for informed, high-risk patients who choose not to accept high-dose IFN- $\alpha$ therapy?

ABSOLUTELY. The authors find the available evidence in favor of IFN- $\alpha$ compelling, but not to the point where informed patients could not make a reasonable decision to forego treatment or seek alternate or investigational therapy. Moreover, the crossover effect observed in the E1690 trial suggests that an untreated high-risk patient who subsequently presents with a resectable recurrence might still derive benefit from adjuvant IFN- $\alpha$, and this should be explained and offered to patients who choose to forego initial IFN- $\alpha$ adjuvant therapy. It must be noted, however, that most contemporary high-risk melanoma patients do not develop resectable recurrences. The widespread adoption of sentinel node biopsy for node staging has resulted in fewer recurrences in regional node basins than the previous practice of clinical staging of nodes. Most contemporary patients with pathologic stage II and stage III disease who develop disease recurrence will not have an option for surgical salvage and subsequent adjuvant IFN- $\alpha$ therapy.

It took nearly 40 years of intensive clinical investigation to develop the first adjuvant therapy regimen with a reproducible and sustained impact on the natural history of high-risk melanoma after surgery. Even in the year 2001, almost all patients who developed nonresectable stage IV melanoma died of their disease. This is a sobering thought, and one that deserves careful consideration in every conversation with melanoma patients regarding adjuvant therapy. As we continue to investigate ways to do better, the small but significant gains made to date should not be discarded lightly. Balancing the pros and cons, adjuvant therapy with high-dose IFN- $\alpha 2 b$ is an important part of the treatment of melanoma. Proponents and opponents of IFN- $\alpha$ should strive to agree on one more thing: let's make the need for adjuvant therapy obsolete by a worldwide commitment to earlier detection and primary prevention!

\section{REFERENCES}

1 Greenlee RT, Hill-Harmon MB, Murray $\mathrm{T}$ et al. Cancer Statistics, 2001. CA Cancer J Clin 2001;51:15-36.

2 Balch CM, Buzaid AC, Soong SJ et al. Final version of the American Joint Committee on Cancer staging system for cutaneous melanoma. J Clin Oncol 2001;19:3635-3648.

3 Frank SJ, Meyers M. Interferon as adjuvant therapy for high risk melanoma. Melanoma Lett 1995;13:1-4.
4 Legha SS. Current therapy for malignant melanoma. Semin Oncol 1989;16(suppl 1):34-44.

5 Steiner A, Wolf C, Pehamberger H. Comparison of the effects of three different treatment regimens of recombinant interferons ( $r$-IFN $\alpha, r$-IFN $\gamma$, and $r$-IFN $\alpha+$ cimetidine) in disseminated malignant melanoma. J Cancer Res Clin Oncol 1987;113:459-465. 
6 Sharfman WH. The role of adjuvant interferon in high risk melamoma patients. Dermatol Surg 2000;26:607-608.

7 Kokoschka EM, Trautinger F, Knobler RM et al. Long-term adjuvant therapy of high-risk malignant melanoma with interferon $\alpha 2 b$. J Invest Dermatol 1990;95(suppl 6):193S-197S.

8 Cascinelli N, Bufalino R, Morabito A et al. Results of adjuvant interferon study in WHO melanoma programme [letter]. Lancet 1994;343:913-914.

9 Cascinelli N. Evaluation of efficacy of adjuvant rIFN $\alpha$ 2a in melanoma patients with regional node metastases. Proc Am Soc Clin Oncol 1994;14:410a.

10 Rusciani L, Petraglia S, Alotto M et al. Postsurgical adjuvant therapy for melanoma: evaluation of a 3-year randomized trial with recombinant interferon- $\alpha$ after 3 and 5 years of follow-up. Cancer 1997;79:2354-2360.

11 Creagan ET, Dalton RJ, Ahmann DL et al. Randomized, surgical adjuvant clinical trial of recombinant interferon alfa-2a in selected patients with malignant melanoma. J Clin Oncol 1995;13:2776-2783.

12 Kirkwood JM, Strawderman MH, Ernstoff MS et al. Interferon alfa- $2 \mathrm{~b}$ adjuvant therapy of high-risk resected cutaneous melanoma: the Eastern Cooperative Oncology Group Trial EST 1684. J Clin Oncol 1996;14:7-17.

13 Kirkwood JM, Ibrahim JG, Sondak VK et al. High- and lowdose interferon alfa- $2 \mathrm{~b}$ in high-risk melanoma: first analysis of intergroup trial E1690/S9111/C9190. J Clin Oncol 2000;18:2444-2458.

14 Livingston PO, Wong GY, Adluri S et al. Improved survival in stage III melanoma patients with GM2 antibodies: a randomized trial of adjuvant vaccination with GM2 ganglioside. J Clin Oncol 1994;12:1036-1044.

15 Kirkwood JM, Ibrahim JG, Sosman JA et al. High-dose interferon alfa- $2 \mathrm{~b}$ significantly prolongs relapse-free and overall survival compared with the GM2-KLH/QS-21 vaccine in patients with resected stage IIB-III melanoma: results of intergroup trial E1694/S9512/C509801. J Clin Oncol 2001;19:2370-2380.

16 Hillner BE, Kirkwood JM, Atkins MB et al. Economic analysis of adjuvant interferon alfa- $2 b$ in high-risk melanoma based on projections from Eastern Cooperative Oncology Group 1684. J Clin Oncol 1997;15:2351-2358.
17 Gonzalez-Larriba JL, Serrano S, Alvarez-Mon M et al. Costeffectiveness analysis of interferon as adjuvant therapy in high-risk melanoma patients in Spain. Eur J Cancer 2000;36:2344-2352.

18 Cole BF, Gelber RD, Kirkwood JM et al. Quality-of-lifeadjusted survival analysis of interferon alfa- $2 \mathrm{~b}$ adjuvant treatment of high-risk resected cutaneous melanoma: an Eastern Cooperative Oncology Group study. J Clin Oncol 1996;14:2666-2673.

19 Kirkwood JM, Agarwala SS. Systemic cytotoxic and biologic therapy of melanoma. In: DeVita VT, Hellman S, Rosenberg SA, eds. Cancer: Principles and Practice of Oncology. Philadelphia: Lippincott, 1993:747-751.

20 Dickler MN, Coit DG, Myers ML. Adjuvant therapy of malignant melanoma. Surg Oncol Clin N Am 1997;6:793812.

21 Veronesi U, Adamus J, Aubert C et al. A randomized trial of adjuvant chemotherapy and immunotherapy in cutaneous melanoma. N Engl J Med 1982;307:913-916.

22 Livingston P. The unfulfilled promise of melanoma vaccines. Clin Cancer Res 2001;7:1837-1838.

23 Santinami M, Maurichi A, Patuzzo R et al. Impact of clinical trials on the treatment of melanoma. Surg Oncol Clin N Am 2001;10:935-947.

24 Haigh PI, DiFronzo LA, Gammon G et al. Vaccine therapy for patients with melanoma. Oncology (Huntingt) 1999;13:1561-1574, discussion 1574 passim.

25 Morton DL, Nizze A, Hoon D et al. Improved survival of advanced stage IV melanoma following active immunotherapy: correlation with immune response to melanoma vaccine. Proc Am Soc Clin Oncol 1993;12:391a.

26 Hsueh EC, Gupta RK, Qi K et al. Correlation of specific immune responses with survival in melanoma patients with distant metastases receiving polyvalent melanoma cell vaccine. J Clin Oncol 1998;16:2913-2920.

27 Sondak VK, Liu PY, Tuthill RJ et al. Adjuvant immunotherapy of resected, intermediate-thickness, node-negative melanoma with an allogeneic tumor vaccine: overall results of a randomized trial of the Southwest Oncology Group. J Clin Oncol 2000;20:2058-2066. 